Mots. Les langages du politique

$72 \mid 2003$

La ville, entre dire et faire

\title{
La réparation, une approche des espaces urbains
}

Mending, an approach to urban spaces

La reparación, un enfoque de los espacios urbanos

Simone Valcke

(2) OpenEdition

Journals

Édition électronique

URL : https://journals.openedition.org/mots/5593

DOI : $10.4000 /$ mots.5593

ISSN : 1960-6001

Éditeur

ENS Éditions

Édition imprimée

Date de publication : 1 juillet 2003

Pagination : 29-43

ISBN : 2-84788-034-8

ISSN : 0243-6450

Référence électronique

Simone Valcke, "La réparation, une approche des espaces urbains », Mots. Les langages du politique

[En ligne], 72 | 2003, mis en ligne le 28 avril 2008, consulté le 23 avril 2022. URL : http://

journals.openedition.org/mots/5593; DOI : https://doi.org/10.4000/mots.5593 


\section{La réparation, une approche des espaces urbains}

Cette étude s'inscrit dans l'idée que la transformation des territoires urbains s'appuie sur des acteurs différenciés, d'horizons professionnels multiples et qui, de ce fait, produisent des discours spécifiques et divers. Parmi cet ensemble discursif, nous privilégions les écrits qui ont pour objet même cette transformation urbaine, autrement dit les discours des acteurs initiateurs des aménagements ${ }^{\circ \circ}$. À travers une lecture interprétative de ces écrits, nous cherchons à comprendre le sens de l'action sur l'espace, explicitée et mise en mots dans les discours afférents, ceci en admettant l'existence d'une relation étroite entre la parole et les réalisations concrètes. On pourra alors suggérer que la production du discours sur la ville est en même temps production de la ville, ce que Marc Rosemberg qualifie de «production discursive des villes» en précisant: «La production discursive des villes n'est pas infondée, elle coïncide avec la production d'espace, ou du moins l'intention de produire de l'espace ${ }^{1}$.

Dans cette perspective, c'est donc cette «intention» d'action sur l'espace, cette vision du devenir de la ville que nous tentons d'analyser à travers le décryptage du discours, inscrit dans les textes qui organisent l'action. Cette conception de la ville en devenir correspond en général à un compromis résultant de la confrontation des différents acteurs concernés par les enjeux urbains: "La ville est appréhendée par des acteurs ordinaires aussi bien que par les professionnels ou les chercheurs de multiples façons qui contribuent à sa richesse. Irréductible à sa matérialité, son

- Économiste, Institut des mers du Nord, Université du Littoral-Côte d'Opale, 21, quai de la citadelle, BP 5528, Dunkerque cedex 1, simone.valcke@wanadoo.fr.

○o Mots étudiés utilisés par les acteurs: accessibilité; attractivité; centralité; dégradation; désenclavement; redéploiement; restructuration; requalification; redynamisation; revitalisation; renouvellement urbain.

1. M. Rosemberg, 2000, Le marketing urbain en question, Paris, L'Harmattan, p. 115. 
caractère urbain s'élabore symboliquement, notamment dans les nombreux discours qui la traversent et qui, la disant, la configurent $»^{2}$.

L'étude approfondie des notions clés identifiées dans les documents d'urbanisme de la Communauté d'agglomération de Boulogne-sur-Mer, sera conduite dans le cadre d'une approche sémantique des textes concernés, avec une démarche portant à la fois sur l'interrogation du sens et également (quoique de façon marginale) sur l'analyse de la fréquence des notions étudiées. Deux textes ont été ici privilégiés, parmi d'autres documents $^{3}$ dont l'examen a montré qu'il ne s'agissait que de documents préparatoires ou documents d'étape, très largement repris dans ces deux textes, ceux-ci apparaissant donc comme nos seuls éléments de référence. Signalons cependant des différences de nature et de contenu, avant de repérer les points communs qui en font un corpus cohérent et relativement homogène.

Le premier, que nous nommerons document I, est le Projet d'agglomération, intitulé Boulogne $2010^{4}$, publié en octobre 1992, à la demande $\mathrm{du}$ district de Boulogne-sur-Mer, qui se présente comme un projet d'orientation générale et évoque les grandes lignes d'une stratégie volontariste pour l'avenir de l'agglomération de Boulogne-sur-Mer. C'est donc une vision globale (le mot vision est d'ailleurs souvent évoqué) envisagée sur le long terme et inscrite dans un contexte régional, et qui est censée servir de référence, d'outil de cohérence globale du développement à venir. Ce document se veut aussi un instrument de mobilisation des acteurs et d'anticipation des mutations.

Le deuxième document, que nous nommerons document II, est le texte du Grand projet de Ville $(G P V)^{5}$ publié en mars 2000 et rédigé par l'Agence d'urbanisme. Il propose, cette fois, une stratégie d'intervention

2. L. Mondada, 2000, Décrire la ville, Paris, Anthropos, «coll. Villes», p. 6.

3. Autres documents :

- DDE 62, 1993, Diagnostic social urbain.

- DDE 62/Service de la politique de la ville, 2000, Diagnostic de l'agglomération de Boulogne-sur-Mer.

- Boulogne Développement (agence d'urbanisme), 1996, Projet de charte de place portuaire de Boulogne-sur-Mer.

- Boulogne Développement, 2000, Amélioration et extension du port de plaisance.

4. S. Duflos, H. Sibille, J. de Courson, G.-L. Rayssac, 1992, Boulogne 2010, Projet d'agglomération, commanditaire: District de Boulogne-sur-Mer.

5. SCET Nord (BETUR) et Boulogne Développement, 2000, Grand projet de ville: présentation synthétique des enjeux et de la stratégie d'intervention. 
détaillée, et présente de façon plus concrète des actions programmées et des projets beaucoup plus précis dans une perspective de court et moyen terme. Le discours est donc plus pragmatique, plus opérationnel, davantage centré sur les enjeux sociaux et localisé sur des zones précises de l'agglomération. Ces zones sont désignées comme «territoires de projet», et définies comme prioritaires pour la mise en place de la politique contractuelle: il s'agit du quartier du Chemin Vert, de celui de Danrémont et du centre ancien, appelé Basse Ville.

Malgré ces différences entre les deux documents, ceux-ci apparaissent en continuité l'un par rapport à l'autre (le premier est explicitement mentionné comme référence dans le second), à tel point que l'on peut avancer l'hypothèse que le document II a pour objectif de concrétiser les grandes orientations envisagées dans le document I. C'est pourquoi il est possible de retrouver dans les deux textes des champs sémantiques identiques et des articulations du vocabulaire similaires. Nous retiendrons ici la notion de champ sémantique conformément à la définition la plus habituelle donnée en linguistique: «Les champs sémantiques sont des champs conceptuels ou notionnels, marquant un domaine d'expérience auxquels on fait correspondre un ensemble structuré de mots $»^{6}$.

\section{Premier champ sémantique: le vocabulaire de la réparation}

Le premier champ, le plus évident, concerne le discours de la reprise ou réparation de l'espace urbain, il est caractérisé par une utilisation intensive de mots préfixés en re-, comme en témoigne la fréquence d'apparition de termes comme restructuration, redéploiement, requalification, revitalisation, reconversion.

On peut associer la forte présence de ce vocabulaire au contexte particulier de Boulogne, ville touchée par la guerre et reconstruite dans les années 50. La structuration du discours, en effet, commune aux deux documents, met en opposition constamment l'état des lieux, le diagnostic d'une situation présente, vue sous un jour négatif (à travers un vocabulaire exprimant la dégradation) et les propositions d'actions à programmer (par le biais du vocabulaire de la reprise) justifiant ces actions. Ainsi,

6. F. Neveu, 2000, Lexique des notions linguistiques, Paris, Nathan Université, p. 16. 
des termes porteurs de valeurs négatives sont systématiquement opposés à des termes signalant la réparation: par exemple les mots dégradation, dévitalisation, sont confrontés à requalification, restructuration.

De façon schématique, on oppose donc le préfixe de-, significatif ici d'un processus de transformation négative, au préfixe re-, qui signifie la reprise de l'existant pour le reconstituer, l'améliorer, mais qui est également à interpréter comme doté de valeurs de retour en arrière, retour au point de départ. La préfixation en de-appliquée à un verbe ou un adjectif a le plus souvent valeur privative, soit pour former un mot de sens contraire (déplaire), soit pour signifier enlever (déshabiller), et exceptionnellement un sens intensif (démontrer). Le préfixe re- est soit itératif (redire), soit à valeur spatiale ou temporelle avec l'idée de retour en arrière (redescendre) soit à valeur intensive (réchauffer $)^{7}$. Cette proximité langagière des deux préfixes $d e$ - et re- est également repérée dans les discours politiques de la droite et de l'extrême droite étudiés par Maurice Tournier: «Parmi les mots-thèmes de l'extrême droite, on entend résonner une ample série négative préfixée en de-: décadence, déchéance, déclin, décomposition, défaite, dégénérescence, dégradation, démission... $\rangle^{8}$ Cet ensemble est mis en relation avec le re-restaurateur qui est relevé par l'auteur, en particulier dans les discours de Maurras: restaurer, rétablir, redressement, ou ceux de Pétain: renouveau, Révolution nationale. Ceci semble suggérer qu'ainsi apposées l'une à l'autre, ces deux figures du discours renvoient à une logique volontariste, qui s'appuie sur une alternative du type destruction / reconstruction correspondant aux oppositions précédemment repérées dans les discours politiques : déclin / redressement, ou encore décadence / restauration.

Par conséquent, se trouve conforté l'intérêt d'étudier dans ces documents, d'une part, la forte présence des mots en re- et de-, et, d'autre part, le système d'opposition qui les met en relation. L'objectif n'est pas tant de travailler sur les mots eux-mêmes, isolés, que de les mettre en relation les uns avec les autres, comme ce sera le cas dans notre deuxième champ sémantique identifié, avec le rapprochement des mots désenclavement / centralité. Malgré le recours à un vocabulaire apparemment technique et apolitique, nous sommes conduite à souligner un enjeu politique probable

7. A. Lehmann, F. Martin-Berthet, 2000, Introduction à la lexicologie: sémantique et morphologie, Paris, Nathan Université, p. 153.

8. M. Tournier, 1997, «De- et Re- dans le même bateau», dans Des mots en politique, Paris, INALF, Klincksieck, p. 62. 
en ce qui concerne notre corpus de base, et donc pas seulement des enjeux urbains.

\section{L'opposition dégradation / redéploiement}

Le vocabulaire de la reprise ne pouvant être appréhendé isolément, tant il apparait mis en relation avec celui de la dégradation, ce dernier doit faire l'objet d'une première approche incontournable. Cette thématique ne peut être abordée qu'en référence à un constat, celui du déclin, essentiellement lié à la crise économique. Le mot dégradation est associé le plus souvent, d'ailleurs, à la situation sociale: «forte dégradation de la cohésion sociale» (p. 17), «forte dégradation du lien social» (p. 40) qui résulte de nombreux handicaps socio-économiques. De plus, il s'agit d'une aggravation de la situation, comme le souligne le document II qui reprend l'évocation de cette perspective négative: «détresse sociale qui s'aggrave», "un quartier socialement en voie de dégradation» titre le paragraphe consacré au quartier du Chemin Vert (p. 18).

Ce vocabulaire s'applique par ailleurs à la dimension matérielle, dans la mesure où il est fait référence à plusieurs reprises au "cadre urbain dégradé» (document I, p. 16, terme souligné par nous, comme dans les citations qui suivent), "dégradation du centre ville et de certaines cités» (p. 20), «importante dévitalisation de l'agglomération» (p. 40). Le terme dévitalisation fait pendant à celui de revitalisation, constamment évoqué par la suite, et suggère un déficit d'animation. La dégradation est aussi associée au cadre urbain dans le document II, dans lequel est décrit longuement «un parc ancien de logement particulièrement dégradé», pour certains ilots du centre ville (p. 22), mais aussi «dégradation des espaces publics» dans les quartiers de logements sociaux et même, "dégradation constante des bardages des façades» (p. 23).

C'est donc à partir de ce constat de dégénérescence générale qu'est proposé un projet qui vise non seulement à enrayer cette évolution négative, mais aussi à réparer, justifiant l'hypothèse de départ, celle d'un espace urbain issu de la période de la Reconstruction, résultat d'un urbanisme dépassé, déshumanisé et porteur de problèmes sociaux: le document II y fait allusion à plusieurs reprises, évoquant «un urbanisme de tours et de barres sans repère ni sens » (p. 13) à propos du quartier Danrémont, «un quartier issu de la Reconstruction» à propos du Chemin Vert. Il est donc certain que ces quartiers, que l'on appelle ici «quartiers de compensation » ou "cités de transition», se sont réalisés dans un contexte particulier, en application des principes de la Charte d'Athènes, et leur 
conception est aujourd'hui perçue comme un handicap, dans le sens où leur dégradation n'est pas seulement matérielle, mais aussi symbolique et sociale, car source de dysfonctionnements évidents.

Les textes insistent alors sur la nécessité d'opérer une véritable conversion pour retrouver la voie du développement, et mettre en œuvre une politique de régénération. Les notions les plus fréquemment convoquées pour exprimer cette reprise s'appliquent à nouveau autant à la matérialité urbaine qu'au développement économique et social. On peut rassembler l'ensemble de ce vocabulaire autour des notions de redéploiement et restructuration. On se trouve en effet face à une multiplicité d'apparitions de cet élément du vocabulaire, le mot redéploiement étant utilisé avec une forte fréquence et en particulier associé à la dimension urbaine dans le document II : celui-ci présente le projet d'un «redéploiement urbain du centre-ville et de son port» (p. 3).

On peut remarquer paradoxalement que ce mot dont le sémantisme est spatial n'est pas emprunté au vocabulaire de l'urbanisme, mais à celui de l'économie: le terme signifie «réorganisation d'une activité économique, ou d'une branche, notamment par l'accroissement des échanges extérieurs » (Larousse). C'est bien une réorganisation spatiale des fonctions urbaines et portuaires sur le territoire de la ville qui est recherchée, et les moyens pour y parvenir sont explicités sous le titre: «Redéploiement urbain du centre-ville autour d'un port, place centrale» (p. 58), ce titre représentant à lui seul un véritable programme.

Ce terme est aussi utilisé dans son acceptation économique à propos des activités portuaires: "modernisation et redéploiement de l'activité pêche », notions liées et proches de celle de restructuration (notion quantitativement forte dans les deux textes), le but étant de redonner à ce secteur une compétitivité lui permettant de redevenir un secteur économique moteur de l'agglomération. Même constat en ce qui concerne l'activité commerciale: «le commerce doit permettre à l'agglomération de redéployer son potentiel d'attractivité» (p. 52). Quant au terme restructuration, il est toujours employé en relation avec le port, que ce soit à propos de l'activité portuaire ou de l'espace portuaire: «La restructuration porte aussi bien sur les outils économiques (réorganisation du secteur) que sur la qualité des espaces publics (quais, terre-pleins, infrastructures)» (p. 58). Dans cette perspective, on constate la fréquente proximité de cette notion avec celle de requalification, elle-même associée aux termes de redynamisation et revitalisation, et toujours proposée comme alternative au processus de dégradation. 


\section{Requalification et mots associés}

On trouve dans le document I: «restructurer et requalifier l'espace portuaire (nous soulignons)», le réaménagement de cet espace devrait conduire logiquement à lui redonner les caractéristiques pour lesquelles il était qualifié (sous-entendu des caractéristiques de dynamisme industriel et commercial), de raviver ses fonctions et qualifications en améliorant sa matérialité, avec une référence qualitative évidente. Plus loin, «la requalification urbaine du centre-ville» (I, p. 19 et p. 20) implique parfois l'amélioration de la qualité architecturale, qui est jugée "médiocre», ainsi que l'aménagement des espaces publics, mais aussi, parfois la dynamique de l'activité commerciale: «requalification urbaine du centre-ville et re-dynamisation de son commerce» (II, p. 3). Le mot est aussi utilisé pour le logement et pour certains quartiers: «Des étapes sur la voie de la requalification», en référence au quartier du Chemin Vert.

Proche de cette thématique, une autre notion peut être rattachée à la précédente, celle de redynamisation. Le mot est cité trois fois dans le même paragraphe (II, p. 2): «redynamisation du commerce» du centre ville, «redynamisation sociale et urbaine» permise par la mise en œuvre du GPV et enfin «redynamiser le développement touristique de Boulogne-sur-Mer». L'emploi de ce terme suggère une logique différente de celle du développement, il implique un effet d'entrainement puisé sur ses capacités propres à produire des effets positifs, à mettre en action une dynamique interne. L'enjeu est tout d'abord économique: c'est celui de la sortie de crise et du retour au développement économique. Dans le document I, ce terme est associé au domaine social: "redynamiser le tissu social boulonnais» (p. 42). Il s'agit en effet de promouvoir des actions d'insertion au sens le plus large: insertion sociale en général, c'est-à-dire par l'emploi, mais aussi insertion urbaine, «en faisant entrer la ville dans ces quartiers périphériques » et inversement en les intégrant mieux à l'espace urbain central.

Enfin, on peut repérer la forte présence d'un autre terme, essentiellement dans le document II : la revitalisation, notion utilisée habituellement dans le sens de réanimation, qui signifie au sens propre: redonner une âme, c'est-à-dire, redonner vie et animation à un ensemble urbain ou à un quartier, ceci selon trois axes envisagés: social, économique et urbain. Cette revitalisation ne peut pas, la plupart du temps être spontanée: elle résulte de la mise en œuvre d'actions complexes qui ne touchent pas seulement à l'amélioration de l'habitat: résorption de l'habitat insalubre, 
«réhabilitation de logements» (on remarquera toutefois que le mot réhabilitation, qui relève du champ de l'urbanisme est très peu cité dans le corpus), mise aux normes des logements et des bâtiments..., mais elle concerne aussi la réimplantation de fonctions traditionnelles ou nouvelles (commerciales, culturelles ...). L'objectif vise un changement d'image: le passage d'une image négative d'un espace dégradé ou dévitalisé à une vision de dynamisme, de vitalité, avec comme modalité, en ce qui concerne le centre-ville, la revitalisation du commerce: "Le cœur commerçant du centre-ville nécessite une revitalisation forte...» (p. 30). Relevons au passage la figure métaphorique du cœur, lieu de la vie, de l'âme, référence anatomique que l'on retrouve à propos du port, "poumon de l'activité économique», fréquemment utilisée dans les écrits d'urbanisme.

Cette première série de notions s'inscrit dans une approche qualitative de ce que l'on pourrait nommer l'intensité urbaine: intensifier la ville, ce serait intensifier le rapport à la ville, en offrir une meilleure acuité perceptible, et finalement, renforcer le sentiment d'urbanité. Cet objectif est particulièrement servi à travers la notion de renouvellement urbain.

\section{Renouvellement urbain}

Dans la présentation du $G P V$, on propose de mettre en place « une stratégie de renouvellement urbain» sous couvert de la Commission «Habitat et renouvellement urbain». On peut en déduire que, dans ce contexte, le renouvellement urbain, dont la fréquence discursive est particulièrement élevée dans ce document, serait une notion institutionnalisée, parce que c'est la dénomination d'une procédure particulière, qui regroupe un ensemble d'actions projetées dans le cadre du $G P V$.

En effet, le renouvellement urbain est aujourd'hui au premier rang de l'actualité politique urbaine: présent dans le titre même de la loi SRU (Solidarité et renouvellement urbain), il se trouve au cœur d'un nombre croissant de discours. Il est le plus souvent défini comme «une nécessité d'action sur des secteurs urbains qui réclament des actions coordonnées, par suite d'évolutions urbaines suscitant des inquiétudes et réclamant une intervention des pouvoirs publics» (p. 10). Il concerne non seulement la modernisation du cadre bâti, mais aussi la création d'une forme urbaine nouvelle (redéfinition du parcellaire ou invention d'un parcellaire nouveau) et enfin l'enrichissement des fonctions urbaines, de la mixité de l'habitat à la diversification des types d'usage. 
Le mot renouvellement est également décliné avec d'autres modifieurs : «renouvellement qualitatif des quartiers d'habitats sociaux»(p.27), «renouvellement du logement social»(p. 28), en notant que ce mouvement de renouveau s'applique au cadre bâti, mais aussi au contenu social de ces territoires. Ceci est explicitement affirmé à travers deux des ambitions affichées pour ces «territoires de projet», qui sont présentées ainsi :

- d'une part, "renouvellement urbain et mixité sociale» (p. 29), le renouvellement étant ici associé aux quartiers périphériques et relié au thème général de la réintégration, réintégration «d'un quartier dans la ville» dans une perspective de valorisation sociale forte: «suivi social», «participation des habitants»...

— d'autre part, «renouvellement du tissu urbain central» qui s'applique, cette fois, au troisième «territoire de projet», la Basse Ville.

Dans cette perspective du renouvellement, et en recourant à une autre méthode d'analyse, celle de la fréquence, on peut repérer une forte représentation des mots renforcement et renforcer (37 fois cités dans le document I, 22 fois dans le document II), ce qui correspond à une fréquence élevée par rapport à d'autres termes étudiés. Ceci peut traduire le fait que certaines actions sont déjà en cours de réalisation (cas des projets de Développement social et urbain), que certaines procédures ou certaines institutions sont déjà en place et qu'il suffit de réactiver leur potentiel d'action. Renforcer signifie donc que l'on doit leur donner plus de force, plus d'efficacité; mais on peut aussi le rencontrer dans le sens de valoriser: «renforcer le potentiel humain» (p. 73) ou encore «renforcer la notoriété externe de l'agglomération »...

Ce vocabulaire de la reprise du tissu urbain et social traduit la volonté de mise en action des décisions et des opérations permettant le retour à une situation meilleure, pour s'opposer aux années de crise et se démarquer d'un urbanisme décalé, inadapté, datant de la Reconstruction, et peut-être aussi en référence au retour à un passé plus glorieux. Par ailleurs, cette rhétorique vise à mieux spécifier un type particulier d'évolution urbaine, le phénomène de la reproduction urbaine, qui transparait habituellement dans la composition du tissu urbain en strates multiples issues des étapes historiques de l'urbanisation, les villes se constituant par sédimentations successives; c'est aussi l'un des sens de l'expression renouvellement urbain, par opposition à l'urbanisme d'extension. Le fondement de cette distinction est que l'on travaille sur l'existant urbain et non sur un espace vierge. Ceci permet de mettre en évidence des différences de pratiques, mais aussi des différences linguistiques. 
Dans le renouvellement urbain, il faut savoir projeter, démolir, aménager et construire de façon concomitante, synchrone, de telle sorte que les actions correspondantes peuvent se rencontrer simultanément, alors qu'en urbanisme d'extension, ces différentes tâches interviennent de manière séquentielle (processus d'ordonnancement). Ce point permet de comprendre la dimension pragmatique et opérationnelle du discours dans le document II. D'autre part, en urbanisme d'extension, les habitants sont virtuels, dans le renouvellement urbain, ils sont réels; d'où la nécessité dans ce deuxième cas de savoir travailler en prenant en compte les occupants (habitants et activités) et d'intégrer leurs interventions dans les prises de décisions, ce qui explique la place non négligeable, dans ce même document, consacrée à la thématique de la participation des habitants.

Le vocabulaire identifié ici comme vocabulaire de la reprise se différencie du vocabulaire du neuf: ville nouvelle, quartier neuf, nouvelle zone d'habitation. Les nouveaux espaces construits étaient auparavant soit des zones détruites, soit des zones nouvellement aménagées (déplacement du lit de la rivière), soit des zones périphériques, alors qu'aujourd'hui, on peut affirmer que l'on refait la ville sur elle-même, les auteurs des documents insistant d'ailleurs sur l'absence de réserves foncières, comme justification absolue du choix de la politique de reprise de l'espace existant et donc de renouvellement urbain.

À l'issue de cette première étape, il semble que l'on puisse esquisser un début d'interprétation. L'énoncé de ces projets sur l'agglomération, qui s'appuie ainsi avec une telle insistance sur cette dichotomie de la dégradation et de la restructuration laisse supposer que ce discours répond finalement à un objectif de communication interne: il s'adresse prioritairement aux acteurs locaux, élus, habitants, techniciens. Il y aurait une dimension communautariste et incitative, expression d'une volonté d'action forte, qui transparait dans ces textes. Ces éléments nous conduisent à explorer un autre champ sémantique qui réfère à la fréquence et au voisinage de certaines notions associant les mots centralité et désenclavement. Ceci devrait nous permettre, en faisant apparaitre à nouveau un système de mise en relation de termes, de confirmer et d'affiner les résultats précédents. 


\section{Deuxième champ sémantique : centralité / désenclavement}

Une lecture attentive des documents fait apparaitre une mobilisation importante et souvent simultanée de ces différentes notions, d'où l'hypothèse qu'ainsi liées les unes aux autres, elles constituent un ensemble thématique significatif, mais, à la différence du premier champ repéré, il s'agit moins ici d'opposition que de renforcement de sens ou encore de lien de causalité entre elles. Notre objectif est donc de repérer et suivre ces termes afin de déterminer, à travers leurs combinaisons, la portée significative de cette proximité. Ce champ sémantique, moins présent dans le document du $G P V$, est perçu exclusivement à l'échelle de la ville de Boulogne-sur-Mer.

La première mention du terme désenclavement (p. 2) se réfère au «désenclavement économique» du port en général, il est ressenti comme une priorité pour l'agglomération et doit se réaliser à travers le développement économique des filières industrielles, commerciales et touristiques, «afin de faire du Port un espace ouvert, de qualité et attractif» (p. 3).

La notion d'attractivité est constamment rattachée à celle de désenclavement, et associée à deux autres notions, celles d'ouverture et de qualité, qui renforcent la signification sous-jacente du désenclavement. En effet, au sens propre, désenclaver signifie «sortir de l'enfermement»: rompre l'isolement d'une région ou d'un territoire, donc se tourner vers l'extérieur (l'ouverture) et se moderniser (la qualité). L'idée dominante est qu'il faut chercher à rompre l'isolement de Boulogne, sur le plan économique pour redonner au site une capacité d'attraction, à la fois au sens d'attirer (faire venir) mais aussi au sens de séduire (plaire), concernant dans le premier cas les entreprises et dans le second les touristes.

Concrètement, ce désenclavement ne peut se réaliser que par une meilleure accessibilité, nouvelle notion liée aux précédentes: «le désenclavement routier doit être accompagné d'un renforcement des liaisons piétonnes et de l'accessibilité interne afin de relier les différents pôles attractifs » (p. 4). Il faut également souligner la place significative dans les textes de la notion de centre, impliquant cette attractivité, ellemême liée à l'urbanité, c'est-à-dire à l'attrait des fonctions urbaines propres au centre ville, fonctions dites centrales. Ainsi pour renforcer sa centralité, l'accessibilité d'une zone ou d'un quartier doit être amé- 
liorée, permettant son désenclavement et renforçant sa qualité et son attractivité. La relation entre ces différentes notions est dès lors évidente, suggérant un véritable cercle vertueux de l'urbanité, si bien que leur proximité textuelle fréquente s'éclaire. Ces mots se renforcent les uns les autres et sont perçus comme totalement interdépendants (chacun désigne la condition de réalisation de l'autre).

Dans le document $\mathrm{I}$, ce thème est encore plus présent, décliné à des échelles géographiques plus nombreuses et plus larges. C'est en effet sur le plan régional tout d'abord qu'est envisagée la nécessité du désenclavement: il s'agit de mieux tirer parti d'une "position géostratégique exceptionnelle au sein de la région Nord-Pas-de-Calais» (p. 8). Le désenclavement est ensuite évoqué à l'échelle de l'agglomération dans sa globalité. Il se présente alors sous la forme d'un désenclavement physique, c'est-à-dire à travers la mise en œuvre de nouvelles structures de communication, par exemple le «désenclavement routier» par l'autoroute A16 (p. 12) ou le «désenclavement ferré» par l'accès en TGV à la gare de Frethun-Calais (p. 13). Ce potentiel de désenclavement doit se transformer en potentiel de développement et, outre son accessibilité améliorée, c'est à nouveau sur une attractivité renforcée que l'accent est mis.

Dans cette dernière perspective, on trouve le mot désenclavement utilisé dans un tout autre registre: en effet, le «désenclavement des mentalités » est appelé en contrepoint d'une situation présente jugée traditionnellement défavorable: «les Boulonnais restent marqués par une culture de l'enclavement» (p. 13), ce qui se traduit par des attitudes de repli sur soi. Le texte du document I se présente lui-même comme un instrument de mobilisation et de renforcement des acteurs boulonnais avec trois objectifs à atteindre: retrouver la confiance dans l'avenir, réhabiliter la détermination à agir, et favoriser le rassemblement des forces.

Cette proximité permanente et régulière des notions d'attractivité et de désenclavement constitue en elle-même un véritable système, en articulant simultanément sous plusieurs aspects la même thématique. On peut schématiser l'armature du discours à partir des mots clés, ainsi repérés et associés de la manière suivante:

- centralité / désenclavement

— attractivité / accessibilité

- qualité / ouverture

On constate que toute la construction discursive des projets est articulée autour de cette combinaison de notions ainsi reliées les unes aux 
autres qui en forment la structure et en fondent la cohérence générale, et celle des projets eux-mêmes.

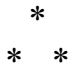

Les mêmes figures linguistiques organisent le discours dans son ensemble: en ce qui concerne le vocabulaire de la réparation d'espaces, par le recours aux préfixations $d e$ - et $r e$-, comme en ce qui concerne les articulations des notions autour de centralité et désenclavement. Cette mise en opposition des termes tend à confirmer la dimension active et volontariste de ces textes: n'est-ce pas le signe qu'il s'agit bien d'un discours constitutif de l'action, au sens ou il est censé préparer des opérations, et également au sens d'un discours mobilisateur? Le vocabulaire et sa mise en forme traduiraient à la fois un engagement et une exhortation à agir, à se ressaisir. De ce point de vue, non seulement le choix du vocabulaire et la fréquence des oppositions ne sont pas neutres, mais ils semblent traduire la force qui agit à travers les mots, ceux-ci devenant alors au sens propre de véritables mots d'ordre émanant des initiateurs de ces projets, en direction des acteurs locaux eux-mêmes.

L'étude de ces documents révèle que tous les concepts repérés et analysés sont utilisés de manière multidimensionnelle, ils sont appliqués simultanément au cadre matériel (cadre bâti et cadre foncier) et à la dimension socio-économique et culturelle. Ceci doit être interprété en relation avec l'évolution même de la conception de la politique urbaine, et en particulier de la politique de la ville (telle qu'elle est concrétisée dans la loi Solidarité et renouvellement urbain) ${ }^{9}$.

Les documents étudiés traduisent d'une certaine manière le changement qui s'est opéré dans cette perspective: longtemps l'aménagement n'a été conçu que sous l'angle des équipements, de la gestion des sols et de l'immobilier (schémas d'infrastructures, Plan d'occupation des sols...). Or, une réflexion s'est engagée depuis plusieurs années sur la nécessité de lier plus étroitement l'occupation du sol avec d'autres politiques publiques, telles que la politique sociale, la politique de transport et de déplacements urbains, celle du logement, et enfin la politique de

9. Ministère de l'Équipement, des Transports et du Logement, 2000, Projet de loi relatif à la solidarité et au renouvellement urbains. 
développement économique en général. Le document du $G P V$ y fait explicitement référence: "Le centre de l'agglomération est le point de rencontre des flux de circulation et d'échanges. S'y mélangent les fonctions économiques, sociales, touristiques et ludiques. Ce redéploiement urbain de la ville est la priorité du projet d'Agglomération et aura un effet d'entrainement en particulier sur l'économie, le tourisme et le commerce» (p. 68).

La charge polysémique du vocabulaire traduit bien ces nouvelles préoccupations à travers l'extension du vocabulaire de l'aménagement spatial à d'autres pratiques et d'autres dimensions. Cette nouvelle orientation institutionnelle inspire le contenu et la tonalité des documents étudiés, au titre même de leur objectif politique et de leur portée mobilisatrice. 